\title{
High-resolution soft $X$-ray spectrometry using the Electron-Multiplying Charge-Coupled Device (EM-CCD)
}

\author{
David J. Hall ${ }^{1}$, James H. Tutt ${ }^{1}$, Matthew R. Soman ${ }^{1}$, \\ Andrew D. Holland ${ }^{1}$, Neil J. Murray ${ }^{1}$, Bernd Schmitt ${ }^{2}$, Thorsten Schmitt ${ }^{2}$ \\ ${ }^{1} \mathrm{e} 2 \mathrm{v}$ Centre for Electronic Imaging, DPS, The Open University, Milton Keynes, MK7 6AA, UK \\ ${ }^{2}$ Paul Scherrer Institut, 5232 Villigen PSI, Switzerland
}

\begin{abstract}
The Electron-Multiplying Charge-Coupled Device (EM-CCD) shares a similar structure to the CCD except for the inclusion of a gain register that multiplies signal before the addition of read-noise, offering sub-electron effective readnoise at high frame-rates.

EM-CCDs were proposed for the dispersive spectrometer on the International X-ray Observatory (IXO) to bring sub-300 eV X-rays above the noise, increasing the science yield. The high-speed, low-noise performance of the EMCCD brought added advantages of reduced dark current and stray-light per frame, reducing cooling and filtering requirements. To increase grating efficiency, several diffracted spectral orders were co-located so the inherent energy resolution of the detector was required for order separation. Although the spectral resolution of the EM-CCD is degraded by the gain process, it was shown that the EM-CCD could achieve the required separation.

The RIXS spectrometer at the Advanced Resonant Spectroscopy beamline (ADRESS) of the Swiss Light Source (SLS) at the Paul Scherrer Institute currently uses a CCD, with charge spreading between pixels limiting the spatial resolution to $24 \mu \mathrm{m}$ (FWHM). Through improving the spatial resolution below $5 \mu \mathrm{m}$ alongside upgrading the grating, a factor of two energy resolution improvement could theoretically be made. With the high-speed, low-noise performance of the EM-CCD, photon-counting modes could allow the use of centroiding techniques to improve the resolution. Using various centroiding techniques, a spatial resolution of $2 \mu \mathrm{m}$ (FWHM) has been achieved experimentally, demonstrating the benefits of this detector technology for soft X-ray spectrometry.
\end{abstract}

This paper summarises the use of EM-CCDs from our first investigations for IXO through to our latest developments in ground-based testing for synchrotron-research and looks beyond to future possibilities.

Keywords: CCD, EM-CCD, soft X-ray, spectrometer, IXO, RIXS

\section{INTRODUCTION}

CCDs have been used on several X-ray astronomy missions since their first use on the Advanced Satellite for Cosmology and Astrophysics (ASCA), launched in $1993^{1}$. With their large area format, high dynamic range, excellent spectral and spatial resolution and low dark current when cooled, they continue to be proposed for future X-ray space instruments.

The Off-Plane X-ray Grating Spectrometer (OP-XGS) that was designed to be flown on the International X-ray Observatory (IXO) is one such instrument. It would have provided an order of magnitude increase in resolution and effective area over the current X-ray spectrometers being flown on XMM-Newton and Chandra ${ }^{2,3}$. To further increase the performance of the OP-XGS, it was decided to benchmark Electron-Multiplying Charge-Coupled Devices (EM-CCDs) as the detectors on the focal plane array.

Through the continued study of EM-CCD technology for X-ray detection that was started with IXO, it was proposed that the high frame rate, high Signal-to-Noise $(\mathrm{S} / \mathrm{N})$ ratio and suppressed readout noise performance of this type of device could benefit other ground-based applications in synchrotron facilities.

*david.hall@open.ac.uk; phone +44 (0)1908 659 579; www.open.ac.uk/cei

UV, X-Ray, and Gamma-Ray Space Instrumentation for Astronomy XVIII, edited by

Oswald H. Siegmund, Proc. of SPIE Vol. 8859, 88590H · (C) 2013 SPIE

CCC code: $0277-786 X / 13 / \$ 18 \cdot$ doi: $10.1117 / 12.2024010$

Proc. of SPIE Vol. $885988590 \mathrm{H}-1$ 


\section{INTERNATIONAL X-RAY OBSERVATORY (IXO)}

The International X-ray Observatory (IXO) was a proposed L-class (large) mission in the ESA Cosmic Vision 20152025 and NASA 2010 Astrophysics Decadal Survey, "New Worlds, New Horizons" studies along with collaboration from JAXA ${ }^{4}$. The mission was designed to investigate the fundamental questions in high energy astrophysics such as black hole formation and evolution, cosmic feedback and finding the missing baryons in the Universe (the Cosmic Web). To achieve its goals, IXO was designed to have a $3 \mathrm{~m}^{2}$ optic and a focal length of $20 \mathrm{~m}$, allowing unprecedented sensitivity, effective area and energy range $(0.1 \mathrm{eV}$ to $40 \mathrm{keV})$. One of the proposed instruments was the dispersive spectrometer that was being competitively bid for by a team proposing a Critical-Angle Transmission (CAT) grating and another team proposing an Off-Plane X-ray Grating Spectrometer (OP-XGS).

\section{DISPERSIVE SPECTROMETER ON IXO}

The Off-Plane X-ray Grating Spectrometer (OP-XGS) on IXO was designed to provide an order of magnitude increase in resolution and effective area over the current grating spectrometers on XMM-Newton and Chandra. The incident photons would be dispersed by a module of off-plane gratings and detected on a CCD array approximately $5 \mathrm{~m}$ away. The gratings would be radial grooved to match the convergence of the beam and blazed to preferentially throw the dispersed X-rays to one side of zero-order. The instrument was designed to give a resolving power $(E / \Delta E)$ greater than 3000 and an effective area greater than $1000 \mathrm{~cm}^{2}$ over the $300 \mathrm{eV}$ to $1000 \mathrm{eV}$ energy range. To achieve this resolution, several dispersed orders were required, therefore different energy photons from different dispersed orders would be detected at the same point on the focal plane; the inherent energy resolution of the CCD is required to separate out these photons ${ }^{5}$.

Initially, a conventional CCD was benchmarked for the focal plane array; however, this decision was changed to use an EM-CCD. The ability to have a higher frame-rate with no increase in readout noise was considered to be more beneficial than the impact that multiplication gain has on spectral resolution.

The high resolution performance of the OP-XGS was provided through the use of an off-plane grating and the position that the photons are detected on the focal plane allows the energy to be measured. At low energies the Quantum Efficiency (QE) of the detector limits the instrument's effective area and so thin filters are necessary to maximise the instrument's performance. The filters are in place to reduce the optical background in the instrument. To counteract the increase in optical background caused by using thinner filters the detectors must be read out quickly; however, this leads to an increase in readout noise.

Through the use of EM-CCDs, the on-chip multiplication gain can be used to suppress the readout noise to sub-electron levels, allowing the fast frame-rate required to minimise optical background. A high frame-rate also reduces dark current generation in the device and so higher flux environments can be observed without pile-up in the pixels. The EM-CCD (Section 4) allows the optimisation of the instrument effective area, but the use of multiplication gain causes a degradation of spectral resolution (Section 5). This degradation could hinder the device's ability to separate out overlapping orders; however, it was found that the degradation was small enough not to be a limiting factor in this instrument (Section 6).

\section{ELECTRON-MULTIPLYING CCD (EM-CCD)}

An EM-CCD takes the architecture of a conventional CCD and adds a multiplication register to the end of the serial register, Figure 1. By accelerating the charge packet through a large potential difference (typically between $40 \mathrm{~V}$ and $50 \mathrm{~V}$ ) the electrons in the charge packet have a small probability (of the order of 0.01) of ionising an additional electron from the silicon lattice through impact ionisation ${ }^{6}$. By repeating this process over several elements (typically 500 to 600 ) it is possible to increase the number of electrons in the charge packet by 100-1000 times.

As this gain process occurs "on-chip", before the readout noise is added to the signal, the readout noise is effectively suppressed allowing a much higher readout speed without an increase in the apparent read noise. However, this multiplication gain process adds another component of noise (similar to shot noise) to the signal due to its stochastic nature. This noise component has been quantified as the Excess Noise Factor for optical photon detection and the Modified Fano Factor when detecting X-rays. 


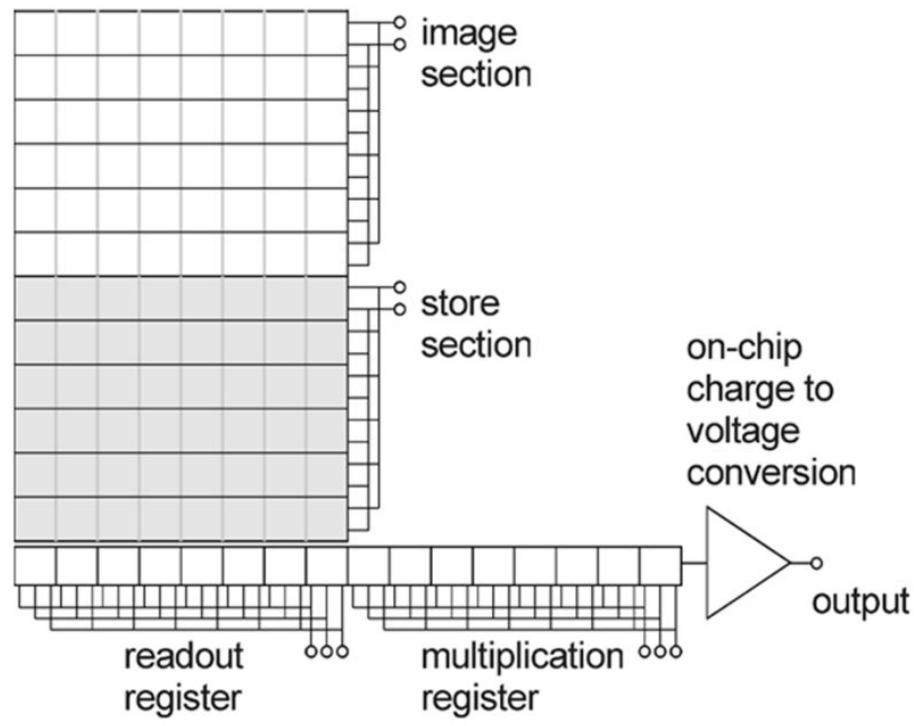

Figure 1. Schematic of an EM-CCD with a multiplication register added between the readout register and the output node ${ }^{7}$

\section{MODIFIED FANO-FACTOR}

The Excess Noise Factor (ENF), the increase in noise caused by detecting optical photons in an EM-CCD, was described by Robbins et al. ${ }^{7}$ for increasing levels of multiplication gain. It was found that at high levels of gain (those greater than approximately 10 times) the combined noise from the photon detection and multiplication gain process was equal to a factor $\sqrt{2}$ higher than that from the shot noise on the detection alone (i.e. the variance on the signal was equal to twice the mean signal level). When the gain of the EM-CCD is equal to 1 the ENF falls to 1 as there is no longer a component of noise from the multiplication register (no gain is applied). The relationship between the ENF and the level of gain is the EM-CCD is given by Equation 1 and shown in Figure 2, where $g$ is the gain per transfer in the register and $G$ the total gain given by $(1+g)^{N}$, where $N$ is the total number of multiplication elements in the register.

$$
\mathrm{ENF}=\frac{1}{G}\left(\frac{2 G+g-1}{g+1}\right) \approx 2 \text { for large } G
$$

The Modified Fano-Factor came from an extension of this work to consider a similar factor for X-ray photons ${ }^{8}$. As the shot noise on the detection of X-rays in an EM-CCD is Fano-limited, the Modified Fano-Factor varies from the Excess Noise Factor by the Fano Factor (0.115 in silicon) at high gains, but is somewhat more complex for lower gain levels. At a gain of 1, the multiplication register no longer adds a noise component to the system; therefore the Modified FanoFactor tends to 0.115 as in the case of a standard CCD. At high levels of gain the multiplication register adds a component of noise that is equivalent to Gaussian shot noise; hence, the Modified Fano-Factor tends to 1.115 in a similar way that the ENF tends to 2. The behaviour of this source of noise between the gain of one and high gain cases is described by Equation 2 and shown in Figure $3^{8,9}$. This effect causes a broadening of the X-ray peak in the detected spectrum, which degrades the spectral resolution of the detector itself, Figure 4. The OP-XGS required a spectral resolution performance from the CCDs that would allow photons separated by $200 \mathrm{eV}$ to be identified separately. It was found that the expected degradation caused by an EM-CCD would not degrade the spectral resolution performance sufficiently to cause problems for the OP-XGS.

$$
\mathrm{F}_{\text {mod }}=\frac{(f G(1+g)-(1-g)(1-G))}{G(1+g)}
$$




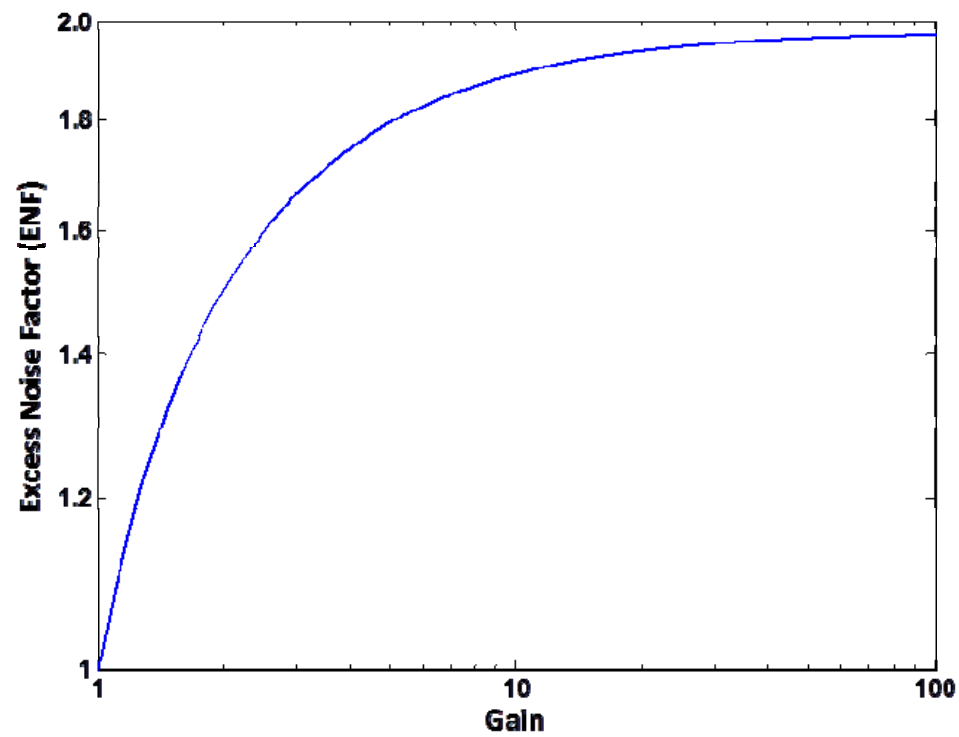

Figure 2. Visible light: The change in the Excess Noise Factor with increasing levels of multiplication gain.

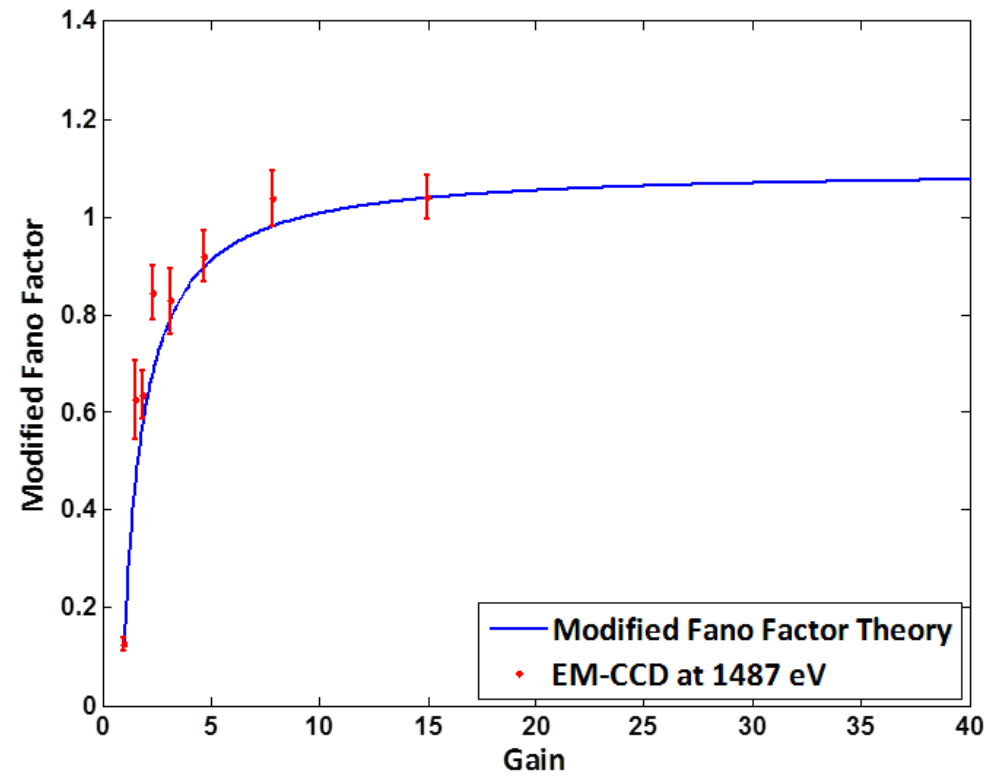

Figure 3. X-rays: The change in the Modified Fano-Factor with increasing levels of multiplication gain. 


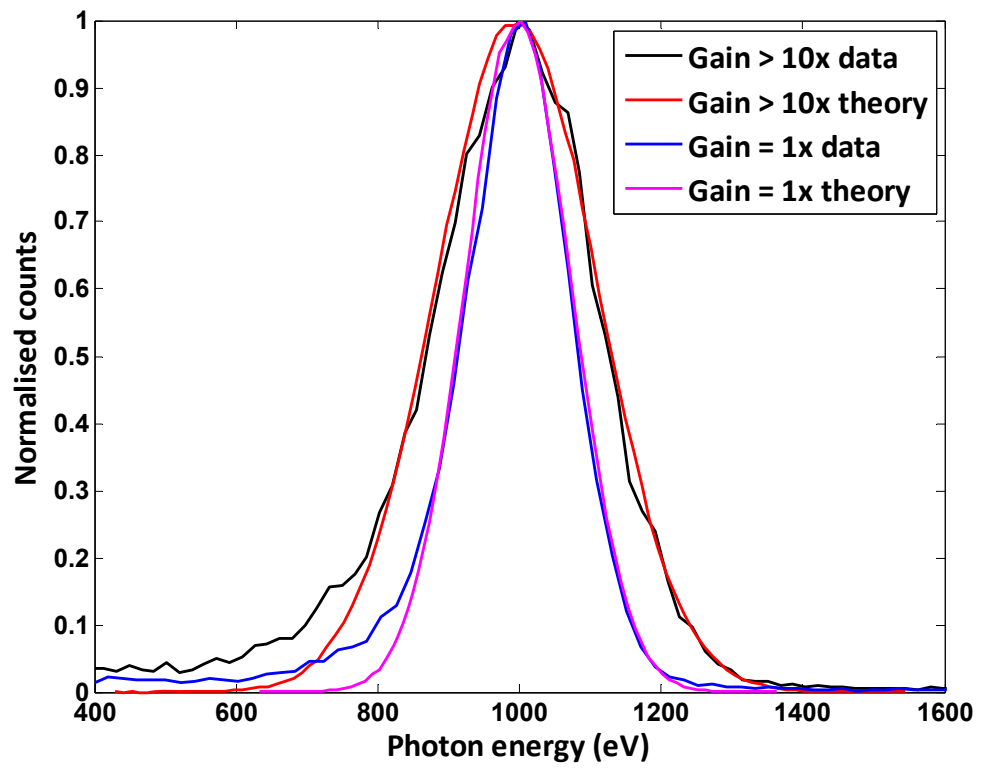

Figure 4. The X-ray peaks predicted using the Modified Fano Factor at $1000 \mathrm{eV}$ compared with real data, taken using the CCD220 with a readout noise of approximately 20 electrons at a gain of 1.

\section{EM-CCDS FOR IXO}

To optimise the scientific performance of the instrument, the detectors used need to be low-noise, high-speed, with high QE over the energy range and good inherent spectral resolution. CCDs are very effective detectors of optical photons which would lead to a stray-light background in the frames taken, limiting the low energy performance of the instrument. To minimise this effect, thin filters would be deposited onto the detectors to block out optical light; however, the Al coatings will cause a drop in the device QE. If the devices can be read out at higher speed then thinner filters can be used and still offer the same level of stray-light blocking which is why a high frame rate ( $\sim 10$ frames/s) would be ideal.

A high frame rate will lead to an increase in readout noise from the detector which harms the instruments performance and this is where using EM-CCDs on IXO offer their first advantage. Multiplication gain causes readout noise suppression and so any increase in this noise source due to high readout speeds can be mitigated.

The higher speed will also mean a reduced level of dark current in each frame. The dark current could be suppressed by cooling the devices; however, an EM-CCD wouldn't have to be as cold as a conventional CCD for the same level of dark current generation per frame.

An additional benefit of high frame-rates is that high flux sources can be observed without having pile-up in the detector (multiple X-ray hits per pixel).

Multiplication gain will allow lower signal events to be easily detected from the background by increasing the Signal-toNoise $(\mathrm{S} / \mathrm{N})$ ratio of the system. This would allow the energy range of the instrument to be increased down to lower energies, increasing the possible science yield of the instrument down into the Lyman forest of fluorescence lines towards UV energies.

To achieve the necessary effective area in the instrument, several overlapping orders need to be used from the grating. If the detector is unable to separate out these lines then it is unsuitable for the application. The Modified Fano-Factor describes the degradation of spectral resolution that occurs when you use an EM-CCD to detect X-rays and through this analysis it has been shown that the degradation caused can be tolerated by the instrument; therefore, the EM-CCD is the perfect candidate for the dispersive spectrometer on IXO. 


\section{GROUND-BASED DISPERSIVE X-RAY SPECTROMETERS}

Following the studies of the EM-CCD for IXO, it became apparent that the EM-CCD would also bring major benefits for ground-based dispersive X-ray spectrometers. Even greater benefits are apparent for applications which are purely dispersive and do not require the inherent energy resolution of the detector to separate differing orders.

The ability to operate the detector at high readout rates, and therefore shorten integration times whilst maintaining sub-electron effective readout noise, opens the door to the use of photon-counting and more advanced analysis techniques. Through the use of photon-counting and the application of centroiding techniques the spatial resolution of the detector can be improved dramatically ${ }^{10-14}$ and as such, the spectral resolution will consequently be improved. One such example of the improvements that are predicted for use of the EM-CCD in ground-based dispersive X-ray spectrometers can be taken from the current research into developments in the camera for the Super Advanced X-ray Emission Spectrometer at the Swiss Light Source (Paul Scherrer Institute).

\section{SUPER ADVANCED X-RAY EMISSION SPECTROMETER (SAXES)}

The Super Advanced X-ray Emission Spectrometer (SAXES), Figure 5, situated at the ADvanced RESonant Spectroscopy (ADRESS) Beamline of the Swiss Light Source (SLS) at the Paul Scherrer Institute (PSI), was designed for high-resolution Resonant Inelastic X-ray Scattering (RIXS) experiments in the soft X-ray regime (400 eV to $1600 \mathrm{eV})$. The energy resolution of the spatially dispersive spectrometer $(E / \Delta E)$ is greater than 12000 at $930 \mathrm{eV}$.

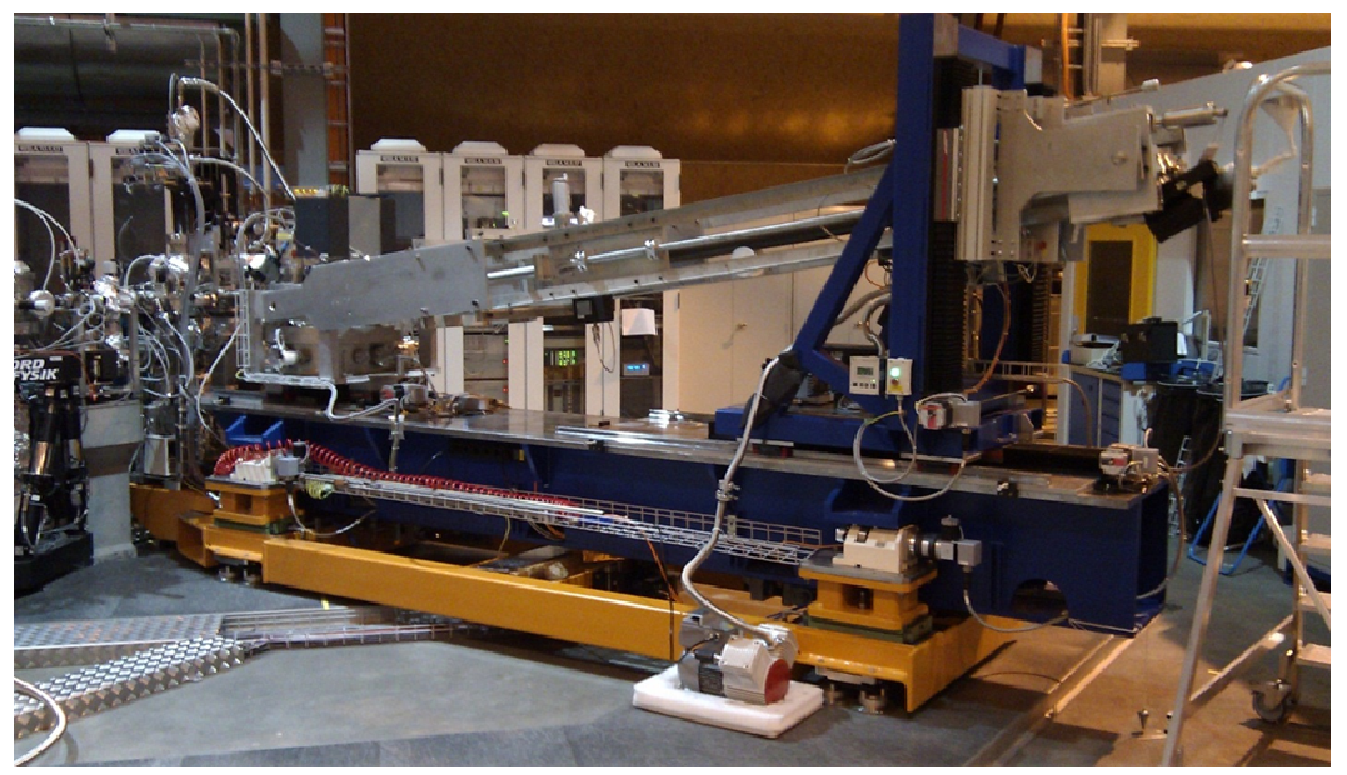

Figure 5. A photograph of SAXES, showing the main arm housing the grating (mid-left) and the black casing surrounding the detector, placed at $20^{\circ}$ to the incident beam (upper-right). The detector system is currently based around a CCD camera.

The spectrometer, SAXES, was designed to use a back-illuminated CCD42-40 ${ }^{15}$, typically operated with an integration time of 10 minutes and pixel readout rate of $100 \mathrm{kHz}$, giving a readout time of approximately 40 seconds and readout noise of a few electrons. To minimise the dark current over the long integration times required to build up sufficient spectral counts, the camera must be cooled, in this case using a liquid nitrogen cryostat to cool the $\mathrm{CCD}$ to $-110^{\circ} \mathrm{C}$.

As the soft X-rays are incident on the back-illuminated CCD, the majority will interact in the field-free region of silicon in the device. In this field-free region, the electrons generated will diffuse radially from the point of interaction until 
they encounter the depleted silicon. In this time, however, the electrons emanating from the point of interaction produce a larger charge-cloud to be collected under the pixels of the device. In the case of SAXES, amongst other aspects of the studies ${ }^{16,17}$, the CCD spatial resolution determined from this charge spreading was measured to be $24 \mu \mathrm{m}$; this spatial resolution directly relates to the spectral resolution achievable with the dispersive spectrometer system.

Through the use of photon-counting and centroiding, an improved spatial resolution of below $5 \mu \mathrm{m}$ has been reported across the energy range of $530 \mathrm{eV}$ to $1000 \mathrm{eV}^{18}$. A reduction in the spatial resolution of the detector to below $5 \mu \mathrm{m}$, coupled with the installation of an improved grating, would allow SAXES to operate with a factor of two improvement in the spectral resolution ${ }^{19}$. However, this experimental result was achieved using a readout rate of $100 \mathrm{kHz}$, meaning that for events to be separable such that they can be centroided, a much shorter integration time would be required and therefore the throughput of the detector would be greatly reduced. Increasing the readout rate would increase the readout noise to a level at which centroiding would not be possible.

Much in the same way that an EM-CCD gave desired improvements for IXO as discussed above, the EM-CCD can also be considered as a candidate for a new detector for SAXES. The EM-CCD would bring the same potential improvements in spatial resolution, and hence spectral resolution, and would reduce requirements for cooling through shorter frame integration times. Alongside these improvements it would also maintain the sub-electron noise that is vital for the most accurate centroid techniques whilst maintaining the throughput.

\section{EM-CCD CAMERA DEVELOPMENT FOR SAXES}

The results reported by Soman et al. ${ }^{18}$ were taken on the PolLux beamline with a variant of the CCD42 that is currently in use in SAXES. The slower readout rate $(100 \mathrm{kHz})$, required to minimise the readout noise and therefore allow the photon-counting centroid algorithms to be applied, would not be conducive to maintaining the spectrometer throughput; much shorter integration times would be required to give spatially separable events in each frame such that the readoutto-integration-time ratio would be too large for efficient spectrometer usage.

Similar experiments were repeated with the $\mathrm{CCD} 97^{20}$, a standard back-illuminated EM-CCD from e2v technologies with $16 \mu \mathrm{m}$ pixels, across four energies: $530 \mathrm{eV}, 680 \mathrm{eV}, 850 \mathrm{eV}$ and $1000 \mathrm{eV}^{21,22}$. With the EM-CCD, it is possible to reduce the readout noise to the sub-electron level through increasing the gain, albeit with an increase in the noise on the signal levels themselves from the associated Modified Fano Factor (Section 5).

At $850 \mathrm{eV}$ and $1000 \mathrm{eV}$, a sub-micron focussed beam of soft X-rays was scanned across a pixel in the CCD97 with results recorded at regular sub-pixel intervals in both the direction of both the columns and the rows of the device. This rastering of the beam provides sufficient data to allow the spread in the calculated centroid locations to be analysed to give a map of the spatial resolution achievable across the sub-pixel grid, Figure 6 and Figure 7. These measurements, as shown in Figure 8, include a certain un-measurable contribution from the width of the beam itself and should therefore be considered as "worst-case" results.

For lower energies, the beam was moved slightly out of focus and rastered in a similar grid pattern, creating an oversampled image of the defocused beam at each energy, Figure 9. By using the beam a small distance away from the focus, the width of the beam can be removed from calculated resolution by de-convolving the known out-of-focus diffraction pattern from the resulting oversampled images.

Through further analysis of these images, it is possible to determine a "worst-case" resolution that has been achieved in these measurements as detailed in Table 1. 

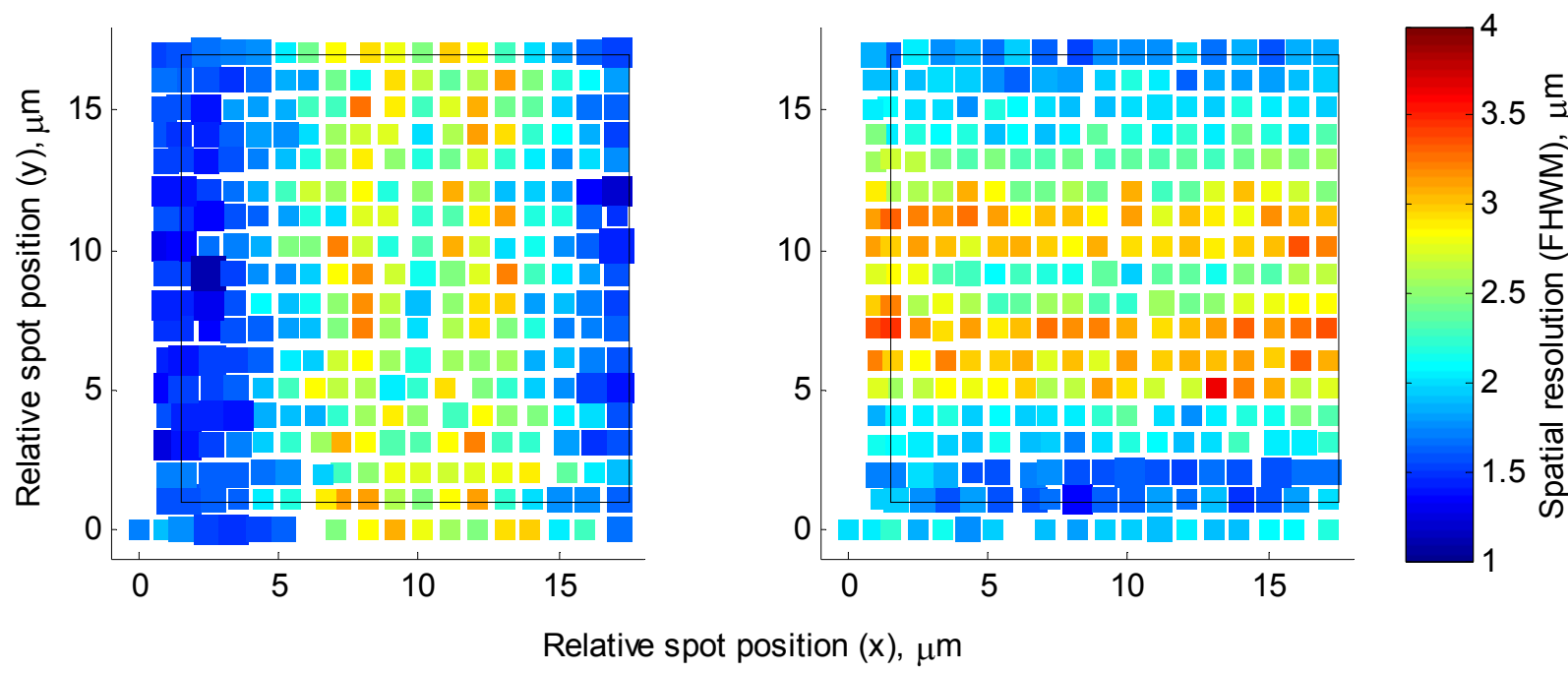

Figure 6. Resolution measured using centroiding of individual X-ray events at $1000 \mathrm{eV}$, in the $x$ (left) and $y$ (right) directions. The location of each data point (small squares) represents the position in the pixel at which the measurement was taken with the pixel boundary shown by the thin black outline. The size of each data point is inversely proportional to the error on the measurement at that point.
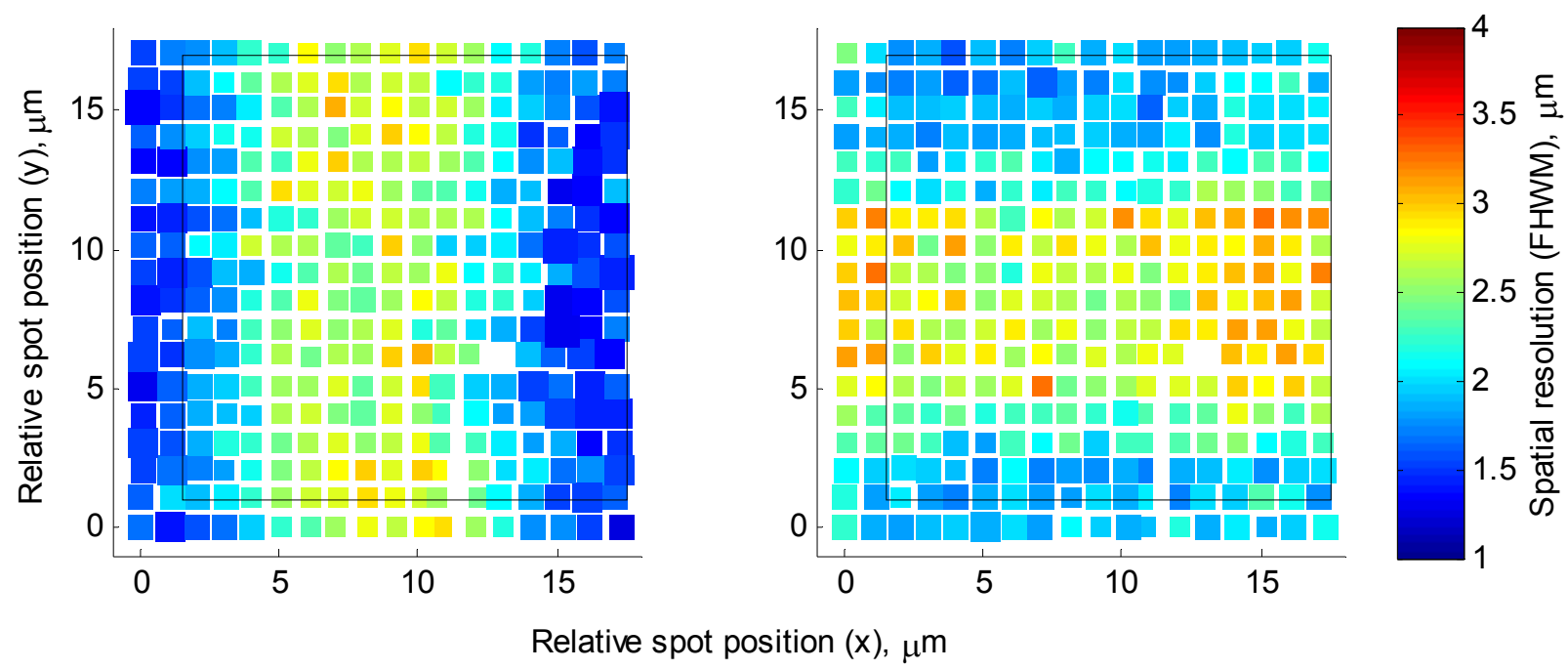

Figure 7. Resolution measured using centroiding of individual X-ray events at $850 \mathrm{eV}$, in the $x$ (left) and $y$ (right) directions. The location of each data point (small squares) represents the position in the pixel at which the measurement was taken with the pixel boundary shown by the thin black outline. The size of each data point is inversely proportional to the error on the measurement at that point. 

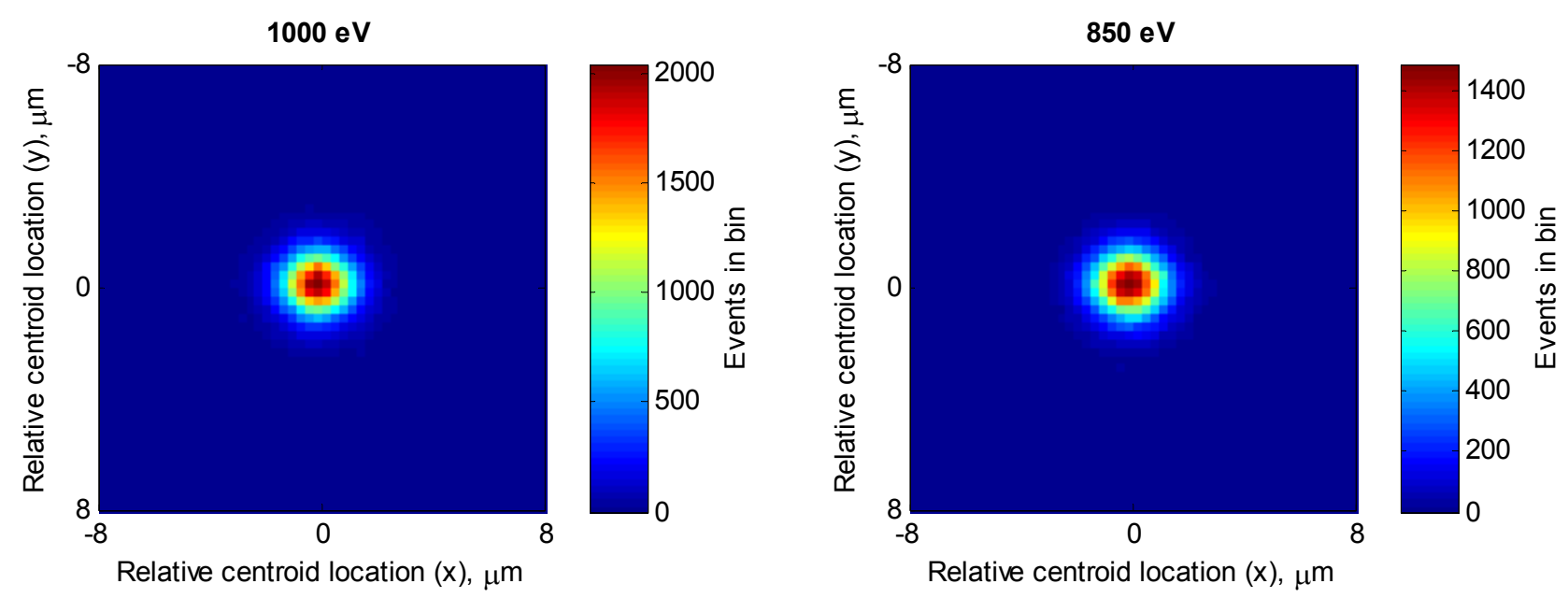

Figure 8. Oversampled centroid location distribution, averaged over all positions at $1000 \mathrm{eV}$ (left) and $850 \mathrm{eV}$ (right), giving a worst-case measurement of the resolution achievable (includes a small component from the beam width). Adapted from Soman et al. ${ }^{21}$.
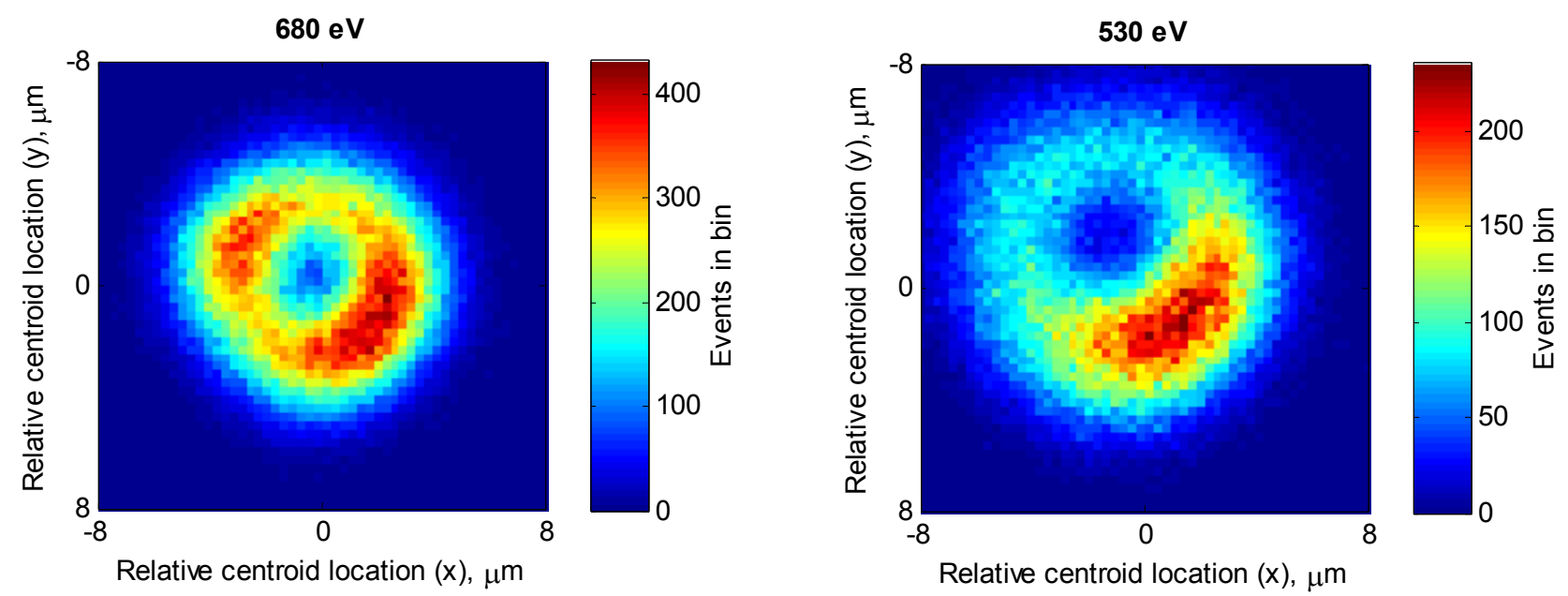

Figure 9. Oversampled centroid location distribution, averaged over all positions at $680 \mathrm{eV}$ (left) and $530 \mathrm{eV}$ (right), here with the beam moved slightly away from the focus to reveal the finer structure from the Fresnel Zone Plate (FZP).

With a known FZP geometry, the beam width can be removed from the images to give a more accurate measurement of the spatial resolution achieved. Adapted from Soman et al. ${ }^{22}$. 
Table 1. Resolution achieved at the four energies measured with the e $2 v \operatorname{CCD} 97^{21,22}$, here showing the de-convolved resolutions for the two lower energies.

\begin{tabular}{|cc|}
\hline $\begin{array}{c}\text { Energy } \\
(\mathrm{eV})\end{array}$ & $\begin{array}{c}\text { Resolution } \\
(\mathrm{FWHM}, \mu \mathrm{m})\end{array}$ \\
\hline 1000 & $2.1 \pm 0.1$ \\
850 & $2.1 \pm 0.1$ \\
680 & $3.0 \pm 0.1$ \\
530 & $3.5 \pm 0.2$ \\
\hline
\end{tabular}

\section{CONCLUSIONS}

Our initial studies into the use of EM-CCDs for soft X-ray detection began with studies aimed towards the IXO mission and the photon-counting, dispersive spectrometer. The promising results of these early studies, alongside the developments of the Modified Fano Factor, showed that the EM-CCD not only provides sub-electron noise performance, but that the ability to run the EM-CCD at much higher frame-rates whilst maintaining the sub-electron noise level allows reduced cooling and filtering to be implemented. Despite the increased noise on the signal, as described by the Modified Fano Factor, the EM-CCD was found to be an ideal candidate for the dispersive spectrometer.

The use of the EM-CCD for soft X-ray detection is not limited to space applications; ground-based research can also benefit from the sub-electron noise performance at fast readout rates. In this study we have taken the example of SAXES at the Swiss Light Source, PSI, in which the EM-CCD has been shown to provide the ideal detector for consideration in any future spectrometer upgrade. The use of the EM-CCD is of course not limited to soft X-ray detection and developments for hard X-ray detection using scintillator-coupled EM-CCDs for synchrotron research are ongoing ${ }^{10-14,23}$.

\section{ACKNOWLEDGEMENTS}

With thanks to e2v technologies plc. for providing the CCDs used in the experimental work and Jörg Raabe and Benjamin Watts for their help during PolLux experimental campaigns. A proportion of this work was performed using the PolLux instrument at the Swiss Light Source, Paul Scherrer Institute (PSI), Villigen, Switzerland.

\section{REFERENCES}

[1] Burke, B. E., Mountain R. W., Daniels, P. J., Cooper M. J. and Dolat V. S., "CCD Soft X-ray Imaging Spectrometer for the ASCA Satellite", IEEE Transactions on Nuclear Science, Vol. 41, No. 1, (1994), 375-385.

[2] Brinkman, A. et al., "The Reflection Grating Spectrometer on board XMM", Proc. SPIE, 2808 (1996) 463.

[3] Weisskopf M. C., Brinkman, B., Canizares, C., Garmire, G., Murray, S. and Van Spreybroeck, P., "An Overview of the Performance and Scientific Results from the Chandra X-ray Observatory", The American Astronomical Society, 114 (2002) 1-24.

[4] Barcons, X., et al., "IXO: The International X-ray Observatory”, ESA Yellow book, Draft 0.4, (2010).

[5] Tutt J. H., Holland, A. D., Murray, N. J., Hall, D., J., McEntaffer, R. L., Endicott, J. and Robbins, M., "A study of Electron-Multiplying CCDs for use on the International X-ray Observatory Off-Plane X-ray Grating Spectrometer", Proc. SPIE, 7732, (2010) 774205.

[6] Jerram, P., Pool, P., Bell, R., Burt, D., Bowring, S., Spencer, S., Hazelwood, M., Moody, I., Catlett, N. and Heyes P., "The LLLCCD: Low light imaging without the need for an intensifier", Proc. SPIE, 4306 (2001) 178-186. 
[7] Robbins M. S. and Hadwen, B. J., The Noise Performance of Electron Multiplying Charge-Coupled Devices, IEEE Transactions On Electron Devices 5 (2003) 1227-1232.

[8] Tutt, J. H., Holland, A. D., Hall, D. J., Harriss, R. D. and Murray, N. J. (2012). "The Noise Performance of Electron-Multiplying Charge-Coupled Devices at X-ray Energies”, IEEE Transactions on Electron Devices, 59(1) $167-175$.

[9] Tutt, J. H., Holland, A. D., Murray, N. J., Hall, D. J., Harriss, Richard D., Clarke, A. and Evagora, Anthony M. (2012). "The noise performance of electron-multiplying charge-coupled devices at soft X-ray energy values. IEEE Transactions on Electron Devices", 59(8) pp. 2192-2198.

[10] Hall, D., Holland, A. and Soman, M., "The influence of electron multiplication and internal X-ray fluorescence on the performance of a scintillator-based gamma camera", Nuclear Instruments and Methods in Physics Research Section A: Accelerators, Spectrometers, Detectors and Associated Equipment, 678 (2012) 64- 77.

[11] Hall, D. J. and Holland, A., "Photon-counting imaging camera for high-resolution X-ray and $\gamma$-ray applications", Journal of Instrumentation, 6 (2011) C01022.

[12] Hall, D. and Holland, A., "High resolution x-ray and $\gamma$-ray imaging using a scintillator-coupled electronmultiplying CCD", Proc. of SPIE, 7449 (2009) 74491G.

[13] Hall, D. J., Holland, A. and Smith, D. R., "The use of automatic scale selection to improve the spatial and spectral resolution of a scintillator-coupled EMCCD", Nuclear Instruments and Methods in Physics Research Section A: Accelerators, Spectrometers, Detectors and Associated Equipment, 604(1-2) (2009) 207-210.

[14] Hall, D. J., Holland, A. and Smith, D. R., "Imaging and spectroscopy using a scintillator-coupled EMCCD”, Proc. of SPIE, 7021 (2008) $70211 \mathrm{Z}$.

[15] e2v technologies plc., "CCD42-40 Front Illuminated AIMO Ceramic Compact Pack", A1A-CCD42-40, Issue 4, April 2003.

[16] Ghiringhelli, G., Piazzalunga, A., Dallera, C., Trezzi, G., Braicovich, L., Schmitt T., Strocov V. N., Betemps, R., Patthey, L., Wang, X. and Grioni M., "SAXES, a high resolution spectrometer for resonant Xray emission in the 400-1600 eV energy range", Rev. Sci. Instrum. 77 (2006) 113108.

[17] Dinardo, M. E., Piazzalunga, A., Braicovich, L., Bisogni, V., Dallera, C., Giarda, K., Marcon, M., Tagliaferri, A. and Ghiringhelli, G., "Gaining efficiency and resolution in soft X-ray emission spectrometers thanks to directly illuminated CCD detectors", Nucl. Instrum. Meth. A 570 (2007) 176.

[18] Soman, M. R., Hall, D. J., Tutt, J. H., Murray, N. J., Holland, A. D., Schmitt, T., Raabe, J. and Schmitt, B. "Improving the spatial resolution of a soft X-ray Charge Coupled Device used for Resonant Inelastic X-ray Scattering", Journal of Instrumentation, 6(11) (2011) C11021.

[19] Hall, D., Soman, M., Tutt, James, Murray, N., Holland, Andrew, Schmitt, T, Raabe, J, Strocov, V N and Schmitt, B., "Improving the resolution in soft X-ray emission spectrometers through photon-counting using an Electron Multiplying CCD", Journal of Instrumentation, 7 (2012) C01063.

[20] e2v technologies plc., "CCD97-00 Back Illuminated 2-Phase IMO Series Electron Multiplying CCD Sensor Datasheet”, A1A-CCD97BI_2P_IMO, Issue 3, May 2004.

[21] Soman, M. R., Hall, D. J., Tutt, J. H., Murray, N. J., Holland, A. D., Schmitt, T., Raabe, J. and Schmitt, B., "Improving the spatial resolution of soft X-ray detection using an Electron-Multiplying Charge-Coupled Device", Journal of Instrumentation, 8(01) (2013).

[22] Soman, M. R., Hall, D. J., Tutt, J. H., Murray, N. J., Holland, A. D., Schmitt, T., Raabe, J. and Schmitt, B., "Developing a CCD camera with high spatial resolution for RIXS in the soft X-ray range", Nuclear Instruments and Methods in Physics Research Section A: Accelerators, Spectrometers, Detectors and Associated Equipment (2013) In press.

[23] Tutt, J., Hall, D. J., Murray, N. J., Endicott, J. and Holland, A. D., "Developing a high-resolution x-ray imager using electron multiplying (EM) CCDs", Proc. of SPIE 8852-17 (2013) In press. 